$\mathrm{XV}$.

Aus dem pharmakolog. Institut in $\mathrm{Z} u ̈ \mathrm{rieh}$.

\title{
Zur experimentellen Pathologie und Therapie der akuten Aorteninsuffizienz.
}

Von

Frr. Zollinger aus Zürich.

(Mit 8 Kurven.)

Die nachstehenden Versuche verfolgten den Zweck, die hämodynamischen Verhältnisse zu erforschen, wie sie nach plötzlichem Entstehen einer Aorteninsuffizienz eintreten, und wie sie allenfalls unter Anwendung von Digitalis sich verändern können. Über den letzteren Punkt liegen bereits Untersuchungen von Cloët ta ${ }^{1}$ ) vor; dieselben beziehen sieh aber nur auf die chronisehe Digitalisbehandlung und berieksichtigen nicht das eigentliche Wesen der jeweiligen Zirkulationsveränderungen, sondern nur den Gesamteffekt nach der anatomischen funktionellen Richtung hin. Die vorliegenden Versuche bezwecken also eigentlich mehr, eine Erklärung für jene Ergebnisse zu versuchen.

Die Hervorrufung der Aortenläsion nach Rosenbach gelingt im allgemeinen, besonders bei Kaninchen, sehr gut, wobei die Sonde am besten in der rechtsseitigen Carotis eingeführt wird. Bei Katzen und Hunden sind die Klappen bedeutend widerstandsfähiger, die Durehstoßnng macht infolgedessen größere Sehwierigkeiten; dabei bewährte sich díe Einführung der Sonde in die linke Carotis als am vorteilhaftesten; dadurch geht man dem Durchstoßen der stark gekrümmten und zähen Aorta, was mir bei einem Hund einmal passierte, am ehesten aus dem Wege.

An meinen 52 Versuchen waren beteiligt: 33 Kaninchen im Gewicht von 2100-4450, 15 Katzen im Gewicht von 2900-4100 und vier Hunde. Ieh verlor ein Kaninchen durch Verbluten aus

1) M. Cloëtta, Dieses Arch. Bd. 59.

Archiv f. experiment. Pathol. a. Pharmakol. Bd. 61. 
dem Sternum, 2 wegen Durchstoßung des linken Ventrikels mit der Sonde, eine Katze an Shok und 1 Hund an DurebstoBung der Aorta.

Die Tiere wurden mit einer Urethan-Morphinmischung narkotisiert; wo absolute Bewegungslosigkeit notwendig war, wurde eine eben hinreichende Menge Curare verabreicht; Hunde und Katzen wurden außerdem meist noch mit Äther narkotisiert. Wir erhielten auf diese Weise bei den of mehr als 2 Stunden dauernden Versuchen recht schöne Narkosen.

Um die in Frage stehenden Veränderungen der Hämodynamik einigermaßen messen und danach ein Gesamtbild der gesetzten Veränderungen konstruieren zu können, versuchte ich mit den verschiedensten Methoden sichere Aufschlüsse über einige Faktoren der Zirkulation zu erhalten. Als solche Einzelgrößen, die analysiert werden sollten, erschienen mir:

1. Die Volumenveränderungen des Herzens.

2. Die Veränderungen der Pulskurve.

3. Die Veränderungen des Blutdrucks.

4. Die Veränderungen der Ausflufmenge.

5. Die aus den obigen Ergebnissen rechnerisch und durch die direkte Messung feststellbare Beeinflussung der Zirkulationsgröße.

I. Volumenveränderungen des Herzens.

Zar Registrierung der Volumenschwankungen des Herzens benutzte ich anfangs die sehon von François Franck ${ }^{1}$ ) und Stefani ${ }^{2}$ ) angewandte Herzkanüle. Nach Eröffnung des Thorax führte ich die Kantile dureh das Pericard bindurch direkt in die jenseitige Brustmuskulatur, so daß das Herz quasi an der Kanüle anfgehängt war. Diese wurde mittelst eines dickwandigen Gummischlauchs mit einem Piston-Recorder vereinigt. Das Pericard diente also als Plethysmograph für die Veränderungen des gesamten Herzens. Die angegebene Methode wurde zu demselben Zwecke auch von Knoll ${ }^{3}$ ) und Johannsson und Tigersted t4) angewandt.

In verhältnismäBig zahlreichen Fällen passierte mir aber das Mißgeschick, daß die Ränder der zarten Pericard-Öffnung nachrissen und der Kanüle nicht innig genug anlagen; die austretende Luft verhinderte selbstverständlich eine genaue Registrierung der Volumenschwankungen. Zudem zeigte sich, daß dem Pericard doch eine

1) Franck: Travaux du laboratoire de Marey III, 1877.

2) Stefani: Academia medico-chirugica di Ferrara $187 \%$.

3) Knoll: Sitz. Ber. d. Wiener Akad. Mathem. Naturw. Gesellschaft, 1890.

4) Johannsson \& Tigerstedt: Skand. Arch. f. Physiologie, 1889. 
gewisse Elastizität zu eigen ist, und daß daher die Spannung desselben durch die Art der Aufhängung von Einfluß auf die Größe der Kurve war: Bei Katzen fand ich das Pericard oft sehr eng anliegend, so daß dann die Kanülenöffnung durch das diastolische Herz leicht verschlossen werden konnte und Unregelmäßigkeiten entstanden. Aus all den erwähnten Gründen wage ich nicht, bestimmte Schlüsse aus meinen auf diese Weise gewonnenen Volumenkurven zu ziehen, und ich kann daher den Anschauungen von Tigerstedt beistimmen, daß das Pericard kein einwandfreier Plethysmograph sei. Immerhin will ich doch hervorheben, daB mehrfach die Volumenschwankungen nach der Durchstoßung kleiner wurden, während das Gesamtvolumen sich dagegen vergrößert hatte. Es wturde dies darauf hindeuten, daß sofort nach der Durchstoßung das Herz sich stärker mit Blut fültt und weniger vollständig entleert.

Weil sich die Kanülenmethode als nicht ganz zuverlässig erwies, versuchte ich es mit der Häkchenschreibung. Diese Methode eignet sich ja sehr gut zur Gewinnung von Kurven des linken Ventrikels. Doch auch hier machten sich mannigfache äußere Umstände störend geltend, z. B. genl̈gte schon eine Temperaturänderung, wie sie durch Auerlampen im Experimentierzimmer erzengt wird, sowie die von dem operierten Tier und den Experimentatoren ausgehende Feuchtigkeit und Wärme, um den Seidenfaden etwas auszudehnen, resp. zu verkürzen und dadurch der Kurve ein ganz anderes Bild zu verleiben, resp. eine Tänschung über die Füllung des Ventrikels zu bedingen. Eine weitere Schwierigkeit bei dieser Methode ist gegeben im Einfluß der Atmung. Wenn durch die Bäkchenschreibung die $\mathrm{Zu}$ - oder Abnahme der absoluten Ventrikelftillung angegeben werden soll, so ist natürlich Voraussetzung, daß das Herz sich stets genau an der gleichen Stelle befinde. Trotz aller Sorgfalt läît sich dies aber nicht immer erreichen, weil es fast unmöglich ist, immer den gleichen Stand der Exspiration zu erzielen. Ich versuchte es dann so, daß die Luft des Blasebalges gemischt wurde mit Sauerstoff, wodurch nur minimale Respirationsbewegungen erzielt werden multen. Aber auch hierbei machten sich die oben erwähnten thermischen Einflüsse störend bemerkbar.

Daß die Sache nicht leicht ist, geht aus den vielen weiteren diesbezüglichen Bestrebungen hervor. So versuchten Roy und A dami 1 ), ferner auch Tigerstedt and Johannsson einen starrwandigen Plethysmographen einzufuhren, der dureh eine Gummimembram, in

1) A dami: British med. Journal 1880 und Philosophical Transactions 1892. 
die ein Loch gebrannt wird, sich abdichtet. Eine Verbesserung hat R othberger ${ }^{1}$ ) angegeben, indem er Rezipienten aus Glas verwendete. Ioh habe auch auf diese letztere Art versucht, die reinen Volumensch wankungen der Kammer aufzuzeichnen; es hat sich aber gezeigt, daß offenbar dureh das Anlegen des Plethysmographen meist die Lage des Herzens zur Aorta etwas verschoben wurde, und daB es dann sehr schwer hielt, die Aorteninsuffizienz glatt, d. h. ohne jede Nebenverletzung hervorzurufen. Sebr häufig wurde dabei das Pericard doch in stärkerem Maße beschädigt, und eine merkliche Blutdrucksenkung war die Folge davon. Immerhin liegt ein völlig gelungener Versuch an einem Hund vor. (Kurve I.)

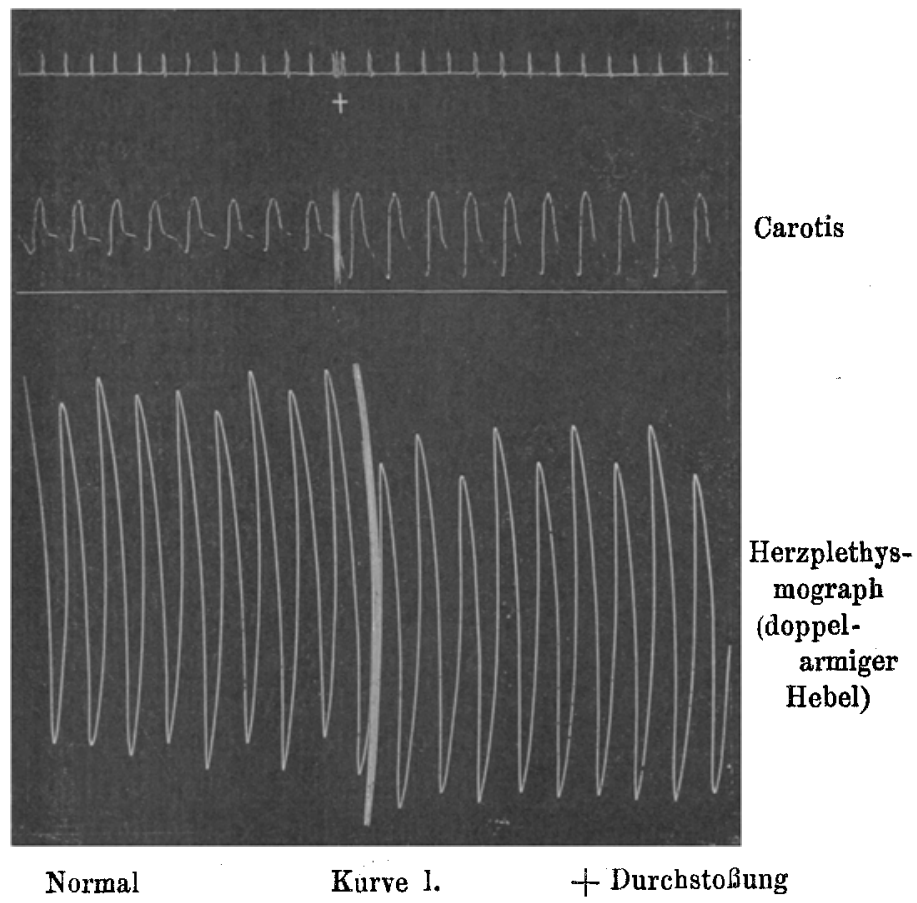

An dieser Kurve läßt sich wiederum deutlich erkennen, wie unter dem Einfluß der Durchstoßung die Kurve in toto etwas nach abwärts rüekt, d. h. das Herz sich etwas vergröBert hat, während die Größe der einzelnen Ausschläge sich kaum verändert erweist. Wenn ich aus diesem letzten einwandfreien Versuch zusammen mit den damit übereinstimmenden anderen Versuchen der Perioard-Plethysmographie einen Schluß ziehen darf, so geht dieser dahin, daß unter

1) Rothberger: Pflügers Archiv, Bd. 118. 
dem Einfluß der Aortendurchstoßung das Herzvolumen sich etwas vergrößert, die einzelnen Schwankungen sich aber gleich bleiben oder etwas kleiner werden. Es kann dies wohl nur so gedeutet werden, dass die Herzkammer sich etwas mehr ausdehnt infolge des zurtickströmenden Blutteils, dagegen sich nicht so vollständig entleert, wie unter normalen Verhältnissen. Immerhin war es nun dringend nötig, diese Ergebnisse durch die weiteren bestimmbaren Faktoren des Kreislaufs za rektifizieren.

Auf die an den genannten etwas unsicheren Größen durch die Digitalis hervorgerufenen Veränderungen möchte ich nicht näher eingehen, da sie keine Beweiskraft beanspruchen könnten.

Die zweite Größe, die ich zur Beurteilung der Verhältnisse verwenden wollte, war die Pulskurve. Diese kann für sich allein ja gar nichts aussagen, aber sie ermöglicht es uns doch, unter Berücksichtigung des Maximal-, Minimal- und Mitteldruckes bestimmte Anhaltspunkte zu gewinnen. Die Resultate sind hier auch viel sicherer und eindeutiger, als bei den entsprechenden Bestimmungen am Menschen, weil durch das Einführen der Kanüle in die Carotis der Druck der Aorta direkt gemessen und die Gefäßwirkungen des gesamten Kreislaufs aufs deutlichste zur Einwirkung kommen müssen, was bei den an der Brachialis oder Radialis des Menschen gewonnenen Resultaten alles nicht zutrifft. Es ist nur noch ein störendes Moment in Rechnung zu setzen als Sicherheitskonstante gegen allfällige Täuschungen, nämlich die Veränderung im Tonus der Gefäße. Wie schon v. Recklinghausen 1) ausgeführt hat, sind die aus der Pulskurve am Menschen gezogenen Schlüsse unsicher, weil wir das Verhalten der peripheren Gefäße nicht kennen, d. b. die Weite des peripheren Reservoirs. Ich glaube aber, daß auch diese Größe in meinen Versuchen wirklich als eine konstante betrachtet werden kann, weil die Tiere sich während des ganzen Versuchs in demselben Stadium niedriger Erregbarkeit befanden, so daß unberechenbare Störungen der GefäBinnervation wohl als gering: angenommen werden durften. Unter Berücksichtigung dieser besonderen Verhältnisse erseheint es mir daher auch erlaubt, aus den Pulskurven Schlïsse zu ziehen, wie man sie bei der unblutigen Sphygmographie am Menschen sicher nicht ziehen dirfte.

Zur Registrierung verwendete ich das Hutrtlesche Torsionsmanometer. Zunächst wurde natürlich bei jedem Tier die normale Pulskurve aufgenommen. Es zeigten sich an dieser schon erhebliche

1) v. Recklinghausen: Arch. f. experiment. Pathol. und Pharm. Bd. 56. 
Schwankungen, sowohl in bezug auf die Größe, also auch namentlich mit Rücksicht auf die Verteilung der verschiedenen Elevationen. Man findet nicht selten Anakrotie, dann namentlich bei Kaninchen oft fast ganz dikroten Puls, daneben alle Übergänge zwischen den beiden Extremen. (Kurve II a, III a, IV a.)

\section{Kurve II.}

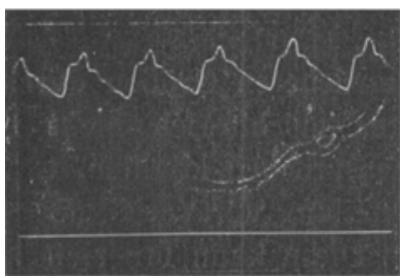

a

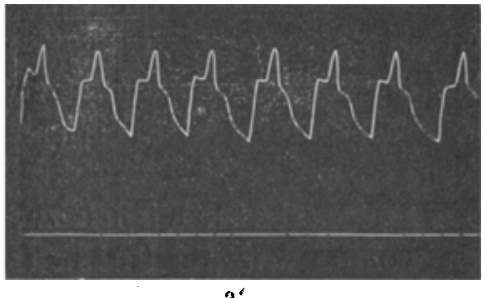

$\mathbf{a}^{\prime}$

Kurve III.

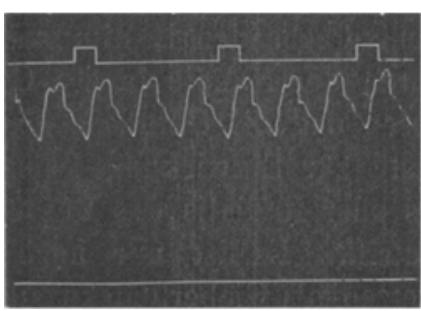

a.

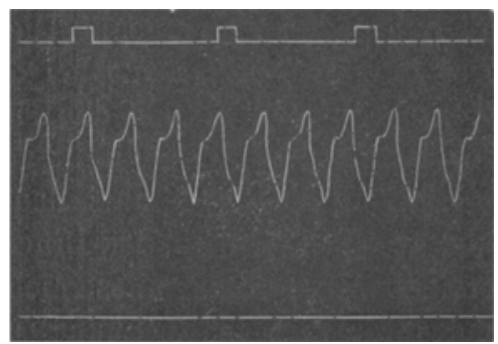

$a^{\prime}$

Kurve IV.

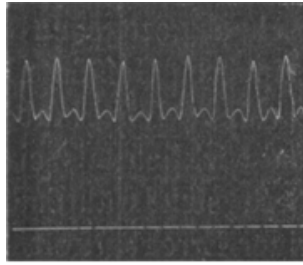

$\mathbf{d}$

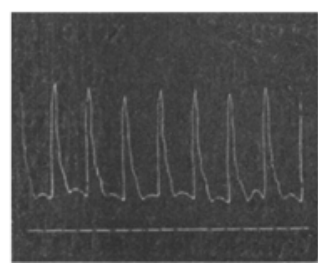

A:

Wie der normale, so bot auch den Insuffizienzpuls kein einheitliches Bild. Einigermaßen erscheint letzterer aber doch von dem vor der Insuffizienz vorhanden gewesenen Pulsbild beeinflaßt zu werden. Zur Illustration hierfür habe ich neben den beiden Typen des normalen Pulses a der Kurve II, III und IV, auch die entsprechenden Aorten-Insuffizienztypen abgebildet, die mit $\mathbf{a}^{\prime}$ bezeichnet sind.

Vollkommen typisch für jeden frisehen Insuffizienspuls war die bedeutende Höhe; aus dieser konnte sofort die gelungene Durch- 
stoßung diagnostiziert werden, was auch schon bei den Kurven I-IV deutlich sicbtbar ist. Als einen ganz besonders instruktiven Beleg hiefür bringe ich in Fig. $V$ die Kure eines Hundes, bei dem neben der leicht als typisch erkennbaren Normalkurve (a) gleich anschließend der Insuffizienzpuls (a') abgezeichnet ist.

Auch hier hat sich der typische Charakter des Hundepulses, d. h. das gegenseitige Verhältnis der verschiedenen Elevationen zu einander, vollkommen erhalten. Es ist dies, nebenbei bemerkt, auch einer der seltenen Fälle, bei denen nach der Durchstoßung eine bedeutende Steigerung des Maximaldrackes eintrat.

Die Ansicht von W. Janowski ${ }^{1}$ ), daß „das Fehlen der RückstoBelevation an der Pulskurve für die typischen und weit fortgesch rittenen Fälle der Aorteninsuffizienz charakteristisch sei". fand

Kurve V.

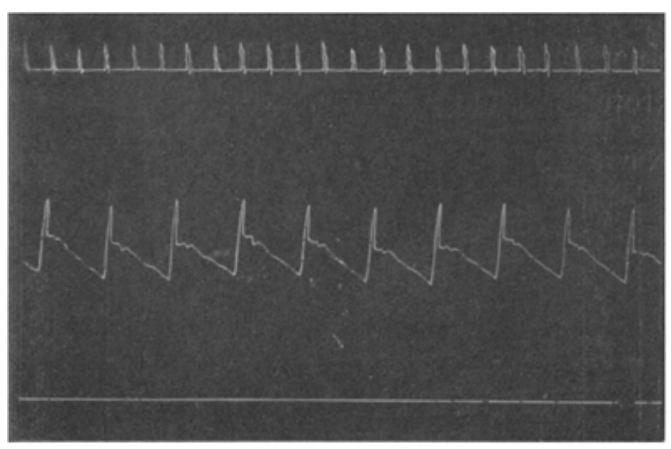

a

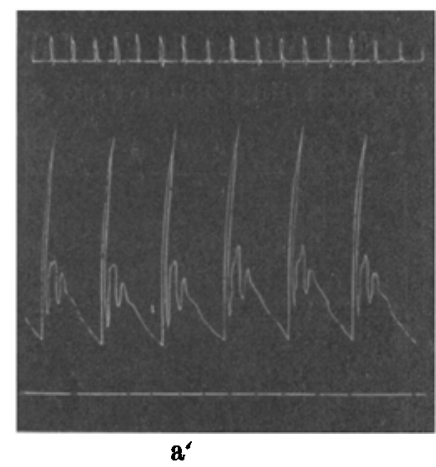

ich in nur etwa der Hälfte der Fälle bestätigt; die andere Bälfte wies die Rückstoßelevation, die sich bis zur deutlichen Dikrotie verstärken konnte, auf. Auch die Ansicht Geigels²) und Goldscheidersi), daß man das Auftreten der Rïckstoßelevation bei Aorten-Insuffizienz mit zur Diagnose eines vitium cordis complicatum, nämlich Aorten-Insuffizienz plus Mitral-Insuffizienz verwenden dürfe, wurde demnach durch meine Versuche nicht bestätigt, da hiebei niemals eine Verletzung der Mitralis stattgefunden hatte. Dabei ist allerdings nicht anßer acht zu lassen, daß es sich in meinen Versuchen um frische Fälle handelte, und daß sie daher nicht ohne weiteres zum Vergleich mit den Erscheinungen herangezogen werden dürfen, wie

1) Janowski, Zeitschr. f. klin. Medizin. Bd. 61 .

2) Geige1, Zeitschr. f. klin. Med. Bd. 61.

3) Goldscheider. Zeitschr. f. klin. Med. Bd. 65. 
sie nach längerem Bestehen des Herzfehlers und eingetretenen Kompensationsvorgängen sich herausbilden.

Neben den oben dargestellten Fällen, bei denen der Insuffizienzpuls den Charakter des Normalen einigermaßen beibehalten bat, kann man aber auch Abweichungen beobachten, bei denen durch die Insuffizienz ein ganz anderes Pulsbild hervorgerufen wird (vgl. die folgenden Abbildungen).

Kurve VI.

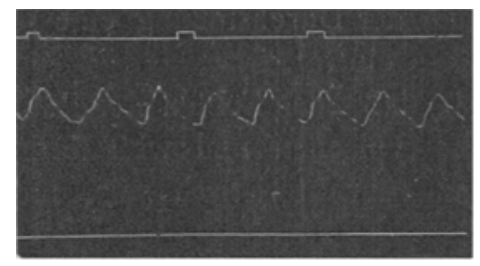

a

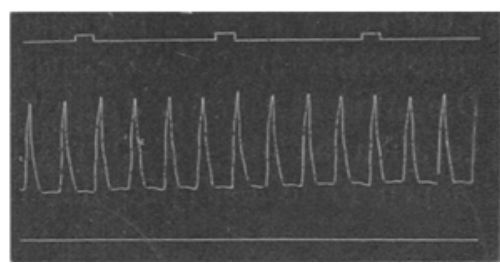

$a^{\prime}$

Fig. VII stellt das Bild einer Durehstoßungskurve dar, bei der sich nach und nach eine anakrote Erhebung entwickelte.

Kurve VII.

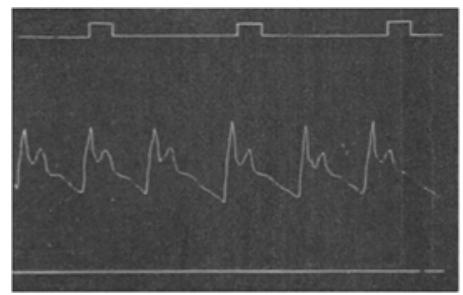

a.

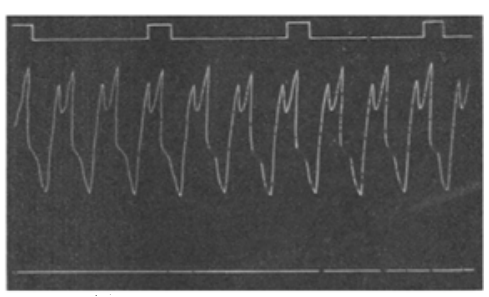

$a^{\prime}$

Über die Ursachen der ziemlich hänfig aufgetretenen Dikrotie bei Aorten-Insuffizienz im Einzelfalle konnte ich mir nicht klar werden; nach den oben schon erwähnten Anschauungen von Goldscheider wäre es schließlich auch denkbar, daß durch das Trauma an der Aortenklappe eine rein funktionelle Störung in der Muskeltätigkeit an der Mitralis erfolgte.

Im Anschlu $B$ an die Besprechung der Pulskurven muß ioh nooh hervorheben, daß im allgemeinen die $\mathrm{Pulszahl}$ sich nicht wesentlich veränderte; es ist dies wohl anch ein Beweis dafür, daß die bereits ansgesprochene Annahme, dureh die Narkose und die künstliche Respiration sei der EinfluB der Nerven auf Herz und Gefäße wesentlich eingeschränkt, ein zutreffender ist. Diese Konstanz kam mir namentlich bei der rechnerischen Bestimmung der Zirkulationsgröße sehr zustatten. 
Zur experimentellen Pathologie u. Therapie der akuten Aorteninsuffizienz. 201

Aus den Beziehungen der Pulskurve zum Blutdruck hat man namentlich in der letzten Zeit versucht, weitgehende Schlüsse auf den Kreislauf zu ziehen. Dieses bei unblutiger Messung unzulässige Vorgehen glaube ich, aus den oben auseinandergesetzten Grinden, bei meiner Versuchsanordnung wohl anwenden zu dürfen.

Es handelt sich dabei namentlich um die Mögliehkeit, aus den Druckverbältnissen der Carotiskurven, wie sie in meinen Versuchen vorliegen, Schlüsse zu ziehen auf die Zirkulationsgröße, speziell auch auf das Schlagvolumen des Herzens. Wie oben erwähnt, haben wir in wenigen Fällen ein Steigen des Maximaldruckes (= systoliseher Druck) beobachtet; sehr hänfig blieb sich der Maximaldruck vor und nach der Durchstobung gleich, in wenigen Fällen nahm er ab. Ganz regelmäßig ergab sich dagegen eine bedeutende Verminderung des diastoliseben Druckes, wie dies ja aucb ein Blick auf die oben abgebildeten Kurven zur Evidenz erweist; von dieser Regel habe ich bei 51 Făllen keine einzige Ausuahme beobachtet. Wenn ich aus meinen Versuchen die Mittelwerte berechne, so erhalte ich

\begin{tabular}{|c|c|c|c|c|c|}
\hline vor der & Durchstoßung & $\mathrm{mm} \mathrm{Hg}$ & nach der & Durchstoßung & $\mathrm{mm} \mathrm{Hg}$ \\
\hline Maximaldruck & Minimaldruck & Pulsdruck & Maximaldruck & Minimaldruck & Pulsdruck \\
\hline 94 & 67,4 & 26,6 & 99 & 41 & 59 \\
\hline
\end{tabular}

Im allgemeinen ergibt sich also nach der Durchstoßung eine Zunahme des Maximaldruckes um 5 Proz. und eine Abnahme des Minimaldruckes um 38 Proz. Die daraus resultierende Zunahme der Amplitude, die ja, wie aus der Tabelle ersichtlich, sich beinahe um 120 Proz. vergrößert hat, ist demnach fast ausschließlich das Resultat der diastoligehen Druekabnahme.

Vergleicht man die Mitteldrucke, die am gedämpften Quecksilbermanometer gemessen werden, vor und nach der Durchstoßung miteinander, so ergibt sich:

vor der Durehstobung $101 \mathrm{~mm} \mathrm{Hg}$. nach der Durchstoßung $77 \mathrm{~mm} \mathrm{Hg}$. d. h. eine Verminderung um ca. 23 Proz. Es stehen diese Ergebnisse im Widerspruch mit den Anschauungen von M. Conto ${ }^{1}$ ), nach welchem für die Aorteninsuffizienz eine Erniedrigung des diastolischen Druckes nicht als charakteristiseh anzunehmen sei. Dagegen stimmen sie tiberein mit den Untersuchungen von $S \operatorname{tra} B b u r g e r{ }^{2}$ ), der auch regelmäßig diese Vergrößerung der Amplitude beobachtete.

1) Conto: Zeitschr. f. klin. Medizin. Bd. 65.

2) Stra Bburger: Zeitschr. f. klin. Med. Bd. 54. 
Bevor ich nun aus den vorstehenden Tabellen der verschiedenen Druckgrößen rechnerisch weitere Schlüsse auf die Zirkulationsgröße, resp. das Schlagvolumen ziehe, möchte ich noch eine weitere Kontrolle durch direkte Messung der während einer bestimmten Zeit ausgetriebenen Blutmenge zur Anwendung bringen. Es wurde zu diesem $Z$ wecke aus einer Carotis während einer gleichbleibenden Anzahl von 1/4 Sekunden durch eine möglichst weite Kanille Blut frei in einen Meßzylinder auslaufen gelassen; das Blut wurde dabei auf normaler Temperatur erhalten und sofort nach der Ablesung wieder in die Vene eingespritzt. Ich weiß wohl, daß ich damit nicht direkt das Schlagvolumen messe; aber es handelte sich ja nur um die Gewinnung von Vergleichszahlen für die Zustände vor und nach der Insuffizienz, und sowohl dafür, wie hauptsächlich auch zur Kontrolle der übrigen Werte erschien dieses Verfabren völlig: ausreichend. Es war dabei auch von Interesse za sehen, wie sich während der Blutentnahme der Blutdruck verhalte. Dies lieferte auch wieder indirekt einen Hinweis auf das Verhalten der Gefäße, resp. auf das Gleichbleiben des Tonus derselben.

Stelle ich nun die auf obigem Wege erhaltenen Auswurfsmengen zusammen, so ergibt sich im Mittel in 10 Sekunden

vor der Durchstoßung

Kaninchen: cem 13,3 nach der Durchstoßung

Katzen:

14,5

cem 10,2

" 12,7

Wir erhalten also eine mittlere Abnahme der relativen Auswurfsmenge infolge der Insuffizienz bei Kaninehen um 23,3 Proz., bei Katzen um 12,4 Proz.

Schon äußerlich gestaltete sich das Ausfließen bei Insuffizienz und in der Norm ganz verschieden; im letzteren Falle ein kontinuierliches Fließen, bei der Insuffizienz ein stoßweises, der Systole entsprechendes Heransschleudern mit einem tropfenweisen Abfließen während der Diastole, also beinahe intermittierend. Diese Erscheinung: ist geradezu ein Sehulexperiment zur Demonstration der Bedeutung des diastolischen Druckes für die kontinuierliche Blutversorgung des arteriellen Systems.

Von verschiedenen Seiten wurde bereits der Versuch gemacht, an relativ einfachen und namentlich auch auf unblutigem Wege zu eruierenden Größen die Auswurfsmenge rechnerisch zu bestimmen. Es hatte deshalb für mich Interesse, an meinen mit sicheren Methoden gewonnenen Werten die Zuverlässigkeit dieser Bestimmungen zu kontrollieren und so indirekt auch einen Beitrag zur klinischen Blutdruokmessung zu liefern. 
Zur experimentellen Pathologie $u$. Therapie der akuten Aorteninsuffizienz. 203

Über die Beziehungen des Schlagrolumens zum Blutdruck spricht sich schon Robert Tigerstedt ${ }^{1}$ ) ans. Aus seinen Versuchen resultiert die Tatsache, daß der Blutdruck an und für sich nicht als $\mathrm{Maß}$ der physiologischen Leistung der Zirkulation gentigt; er fand, daß die vom Herzen herausgetriebene Blutmenge bei einem und demselben arteriellen Druck innerhalb sehr weiter Grenzen variieren kann. Seine Angaben wurden durch meine Versuche in allen Riehtungen bestätigt. Die Tatsache, daß der höchste Blutdruck nicht immer der größten Ausflußmenge entspricht, möchte ich im Ansehluß an Tigersted damit in Zusammenhang bringen, daß das Herz bei starkem Widerstand in den Gefäßen nur mit Schwierigkeit sich entleert. Für jedes Tier existiert ein bestimmter, nicht zu hoher, aber auch nicht zu tiefer Druck, bei dem die größte Blutmenge ausgeworfen wird.

Durch die Blutentnahme wurde natürlich der Blutdruck jeweilen recht bedeutend verringert. Bei den 7 einschlägigen einwandfreien Versuchen betrug der Maximalblutdruck in der Norm vor der Blutentnahme im Mittel $97 \mathrm{~mm} \mathrm{Hg}$, nach der Blutentnahme $66 \mathrm{~mm} \mathrm{Hg}$; die Abnahme beträgt 32,9 Proz. Nach der Insuffizienz sank er bei der Blutentnahme von 102 anf $67 \mathrm{~mm} \mathrm{Hg}$, also um 34,3 Proz. Es ergibt sich somit, daß die Aorteninsuffizienz von einem ganz unbedeutenden Einfluß auf die Abnahme des Blutdruckes bei der Blutentnahme war, daß also während der Experimente der Gefäßtonus sich nicht wesentlich geändert hat.

Fì rst und Soetbeer ${ }^{2}$ ) stellen ein Produkt von Pulsdruck und mittlerem Blutdruck als Maß des Sehlagvolumens auf. Wenn ich diesen Quotienten in meinen Versuchen berechne, so ergibt sich als Mittel vor der Insuffizienz die Zahl 2664, nach der Insuffizienz aber 5991, also eine Zunahme um 125 Proz.

Von denselben Autoren stammt der rein empiriseh gefundene Quotient $\frac{p}{d+\frac{p}{3}} \cdot{ }^{3}$ ). Meine Versuche ergaben einen diesbeztuglichen Mittelquotienten von 0,32 im Normalzustand, einen solehen von 1,26 bei Aorteninsuffizienz; die Zunahme beträgt 290 Proz.

Andere von diesen Autoren vorgeschlagene Quotienten sind $\frac{p}{d}$ und p.n., wobei n die Pulsfrequenz bedeutet. Letzterer Ausdruck soll als

1) Tigerstedt, Ergebnisse der Physisologie, Jahrg. IV.

2) Fürst und Soetbeer, Deutsches Arch. f. klin. Med. Bd. 90.

3) $\mathbf{p}=$ Pulsdruck, $\mathrm{d}=$ diastolischer. Druck. 
"relatives Maß der pro Minute geleisteten Herzarbeit" gelten. Bei der Prüfung der genannten Quotienten ergab sich bei mir eine Zunahme von $\frac{p}{d}$ bei Insuffizienz um mehr als das Zehnfache; $p$. n. wuchs um 94 Proz.

D. Passavant ${ }^{1}$ ) stellt die Herzarbeit als Produkt des Minutenvolumens und des Aortendruekes dar; 0 . Franck bezeichnet diese Berechnungsweise als unberechtigt. Wenn ich bei meinen Versuchen die Herzarbeit nach Passavant berechne, so ergibt sich bei Insuffzienz eine durchschnittliche relative Abnahme gegenüber der Norm um 49 Proz., während die Blutmenge sich nur um 23,3 Proz. verminderte.

Versuchen wir nun, aus den erhaltenen Werten uns ein Bild über die Veränderungen zu machen, die die Herzarbeit, resp. die Zirkulation, durch die Aorteninsuffizienz erleidet, so ergibt sich der Maximaldruck bleibt gleich oder steigt um einen geringen Wert (durchschnittlich 5 Proz.); der Minimaldruek sinkt um 38 Proz., der Mitteldruck um 12 Proz.; der Palsdruck vergrößert sich bedeutend (126 Proz.); die direkt gemessene Auswurfsmenge des Blutes verringert sich um 23,3 Proz. Das Plethysmographenbild zeigt uns nach der Durchstoßung eine in toto etwas nach abwärts geschobene Kurve, was den Schlub rechtfertigt, daß das Herz sich etwas vergröBert, während die Größe der einzelnen Ausschläge keine Veränderung erfahren hat.

Aus dieser Zusammenstellung der Resultate ergibt sich, daß das Herz während der Diastole stärker gedehnt wird. Diese Dehnung bildet wohl einen Reiz für die Muskulatur, wobei ich nur die allgemeine muskelphysiologische Tatsache im Auge habe, nicht etwa die Goltz-Schiffsche Annahme von der Reizwirkung des einströmenden Blutes als Automatie-Erzeuger. Das Herz zieht sich kräftig zusammen, wobei wohl wegen der erhöhten Spannung im Innern des Ventrikels der auf die isometrische Zuckung entfallende Anteil wesentlich kleiner ausfallen diurfte. Um so größer muß dann die Kraft im Gebiete der isotonischen Zuckung sein, und hiebei scheint das Herz seiner Aufgabe nicht mehr völlig gewachsen zu sein, insofern als es nicht vermag, sich vollständig zu entleeren. Aber auch in den Fällen der völligen Arbeitsleistung ist die letztere fast als eine überflüssige, eine Sisiphusarbeit zu betrachten, weil ja stets eine bestimmte Menge Blut während der Diastole zurïckflieBt, und deshalb trotz aller systolischen Anstrengungen der diastolische Druck und das kontinuierliche Fließen nicht aufrecht erhalten werden können.

1) Zit. n. R. Tigerstedt, Ergebnisse d. Physiol. Jahrg. 6. 
Zur experimentellen Pathologie u. Therapie der akuten Aorteninsuffizienz. 205

Der zweite Teil der Arbeit befaBt sich mit dem Studium der Veränderungen, die die eben geschilderten bämodynamischen Verhältnisse nach Verabreichung von Digitalis erfahren. Zur Verwendung gelangte hiebei ein titriertes Infus, ein Dialysat und Digalen, die alle in äquivalenter Menge, durchschnittlich $0,5 \mathrm{mg}$ Digitoxin pro Dosis, nach Eintritt der dureh die Durchstoßung bedingten Veränderungen intravenös injiziert wurden. Bei Hunden wurden entsprechend größere Gaben, bis zu $1 \mathrm{mg}$, bei Katzen meist noch kleinere verabreicht, da ich Wert darauf legte, die Dosierung möglichst innerhalb der nicht toxischen Dosen für die einzelne Tierart zu wählen.

Kurve VIII.

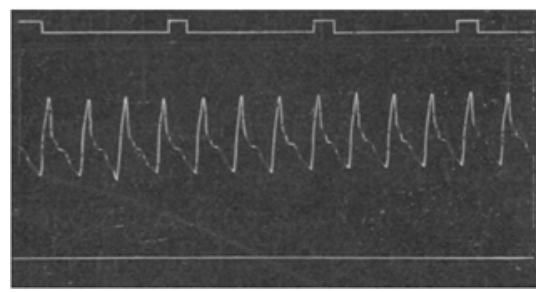

a

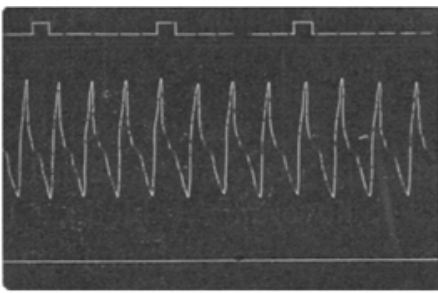

b

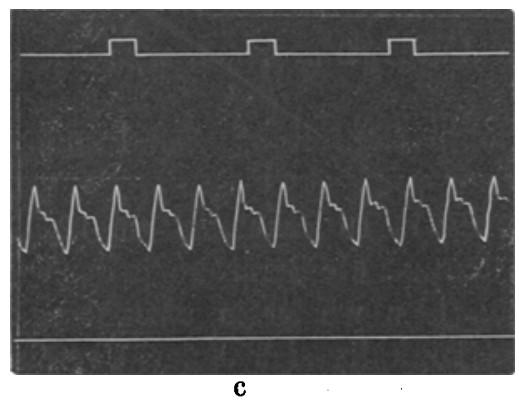

Die einzelnen Präparate unterschieden sich in ihren Wirkangen nicht voneinander. Auch hiebei berücksichtigte ich die Volumenveränderungen des Herzens, die Veränderungen von Pulskurve, Blutdruck und Ausflußmenge.

Die mit der Plethysmographenmethode erhaltenen Resultate scheinen mir zu sehr in der Luft zu hängen, als daß ich es wage, bestimmte Schltusse auf die Volumenveränderungen des Herzens zu ziehen.

Die Verabreichung von Digitalis war nicht imstande, die Puls $k u r v e$ regelmäßig in ihrer Größe beträchtlich zu verändern; im allgemeinen machte sich aber die Tendenz einer Annäherung an die normale Größe bemerkbar. In einigen wenigen Fällen beobachtete 
ich, daB auch die Form sich entsprechend dem normalen Pulsbild etwas änderte. Ein Beispiel hiefür bietet Kurve VIII.

Die Kurven entstammen alle dem gleichen Versuch; Fig. VIII a stellt die Normalkurve, Fig. VIII b die Insuffizienz- und Fig. VIIIe die Digitaliskurve, aufgenommen 70 Minuten nach der Injektion, dar. In anderen Fällen kam es vor, daß nach der Verabreichung des Medikamentes plötzlich eine anakrote Welle sich bildete, die nachher wieder verschwand.

Über die Druckveränderungen gibt folgende Tabelle der Mittelwerte Aufsehluf.

\begin{tabular}{|c|c|c|c|c|c|}
\hline \multicolumn{3}{|c|}{ Insuffizienz ohne Digitalis $\mathrm{mm} H \mathrm{~g}$} & \multicolumn{3}{|c|}{ Insuffizienz mit Digitalis $\mathrm{mm} \mathrm{Hg}$} \\
\hline Maximaldruek & Minimaldruck & Pulsdruck & Maximaldruck & Minimaldruck & Pulsdruck \\
\hline 99 & 41 & 59 & 100 & 48 & 52 \\
\hline
\end{tabular}

Im allgemeinen ergibt sich also eine ziemlich bedeutende Vermehrung des diastolischen Druckes (17 Proz.), während der Maximaldruck unverändert bleibt. Der Pulsdruck zeigt eine Abnahme um 12 Proz., die wiederum ganz zu Lasten der diastolischen Druckänderung fällt; der Mitteldruck weist dementsprechend ebenfalls eine Vermehrung auf.

Stelle ich die Ausflußmengen zusammen, so ergibt sich im Mittel

bei Insuffizienz ohne Digitalis

\section{$10,2 \mathrm{cem}$}

bei Insuffizienz mit Digitalis

$$
11,5 \mathrm{ccm}
$$

also eine Vermehrung in 10 Sekunden um 12,7 Proz.

Ich verzichte darauf, die Berechnung der Quotienten von Fürst und Soetbeer etc. hier wiederum anzuführen, da auch nach der Verabreichung von Digitalis sich keine Übereinstimmung mit den direkt gefundenen Werten des Ausflußrolumens zeigte. Es beweist dies, wie anßerordentlich vorsichtig man sein muß mit der Aufstellung solcher Berechnungen. Sie stimmen meistens nur furr ganz bestimmte Versuchsbedingungen, dulden keine Übertragung auf andere normale Verhältnisse und versagen völlig bei den pathologisehen Zuständen der Ventilstörungen. Ich möchte das besonders betonen, weil in manchen klinischen Arbeiten die Neigung besteht, aus so einfachen Größen wie dem Amplitudenfrequenzprodukt die weitgehendsten Schlüsse auf den Kreislauf zu ziehen. Ich halte diese letzteren in vielen Fällen fur durchaus irreführend ${ }^{1}$ ).

1) Vergleiche hierüber die mir leider erst nach Abschluß der Arbeit zu Gesicht gekommene Abhandlung von 0 . Müller. Ergebnisse d. inn. Med. und Kinderheilkd. Bd. II. 
Zur experimentellen Pathologie u. Therapie der akuten Aorteninsuffizienz. 207

Während der Maximaldruck bei der Blutabnahme bei Insuffizienz von 102 auf $67 \mathrm{~mm}$ Quecksilber, also um 35 Proz. sank, betrug er nach der Injektion von Digitalis vor der Blutentnahme durchschnittlich 100, nachher $60 \mathrm{~mm} \mathrm{Hg}$., die Abnahme beläuft sich auf 40 Proz. Es besteht also in dieser Richtung, namentlich wenn man die um 12,7 Proz. vermehrte Ausflußmenge nach Digitalis berüeksichtigt, gar keine Differenz.

Um einen Beitrag zu liefern zu der vieldiskutierten Frage, ob die unter der Einwirkung von Digitalis erseheinenden Druckveränderungen von der vermehrten Herztätigkeit allein herrühren, oder ob dabei auch die Gefäßmuskulatur im Sinne einer erhöhten oder verminderten Kontraktion eine Rolle spiele, zog ich auch den Widerstand in den Kreis der Betrachtung.

R. Tigerstedt ${ }^{1}$ ) schlägt, allerdings mit Reserve, vor, für den Widerstand den Quotientien $\frac{\mathrm{h}}{\mathrm{q}}=$ Blutdruck dividiert durch Minutenvolumen, zu wählen und bemerkt hiezu: „Wenn wir in einem Versuche finden, daß der Quotient $\frac{b}{q}$ zunimmt, so würde dies bedeuten, daß der Gesamtwiderstand zugenommen hat." $\mathrm{R}$ oy und $\mathrm{Ad} \mathrm{ami}^{2}$ ) fanden in Übereinstimmung der Resultate von Basch und Moll, daß bei vermehrtem Widerstand im großen Kreislauf eine vermehrte Stauung im Herzen auftritt, dessenungeachtet aber keine Veränderungen der von der linken Kammer herausgetriebenen Blutmenge erscheint.

Tigerstedt spricht sich in dieser Hinsicht sehr vorsichtig aus; bei geringer Erhöhung der Widerstände konnte er eine Vermehrung, bei stärkerer Erhöhung eine Verminderung der Ausflußmenge konstatieren. In meinen Versuchen hat sich unter Benutzung des Tigerstedtschen Quotienten und der relativen Ausflußmenge folgendes ergeben:

$\begin{array}{lccc} & & \text { Insuffizienz } & \text { Insuffizienz } \\ & \text { Norm } & \text { ohne Digitalis } & \text { mit Digitalis } \\ \text { Widerstand } & 7,3 & 7,8 & 7,9 \\ \text { Ausflußmenge } & 13,3 & 10,2 & 11,5\end{array}$

Demnach war also bei Insuffizienz der Widerstand um 6,4 Proz. gewachsen; die Darreichung von Digitalis vermochte nicht, ihn merklich zu verändern. Innerhalb der einzelnen Versuche

1) R. Tigerstedt, l. c. $299 \mathrm{ff}$.

2) Roy und Adami, referiert nach Tigerstedt. 
verursachte das Medikament aber bei der ersten Blutentnahme meistens eine oft nicht unbedeutende Erhöhung des Quotienten (durchsehnittlich 35 Proz.). Bei späteren Blutentnahmen sank der Widerstand aber wieder, so daß sich der durchschnittliche Quotient nur ganz wenig höher hielt als derjenige bei Aorteninsuffizienz. Es würde dies also darauf hindeuten, daß nur in einem ganz kurzen Stadium der Digitaliswirkung eine vorübergehende Gefäßkontraktur eintritt, indem bei gleichbleibender Ausflußmenge der Quotient sich nur kurze Zeit erhöhte und nachher zum friheren Stand zurückkehrte. Wenn wir nun diese Tatsache verwerten dutrfen, so ergibt sich der Schluß, daß das durch die Darreichung von Digitalis erreichte höhere AusfluBvolumen zum größten Teil auf Rechnung einer vermehrten verstärkten Herztätigkeit zu setzen ist, daß die Widerstände hiebei nur eine ganz geringe $̈$ Äderung im Sinne einer rasch vorübergehenden Erhöhung erfahren. Es würde dies dafür sprechen, dab wohl im allgemeinen die Digitaliswirkung innerhalb der therapeutisohen Dosen sich hauptsächlich an das Herz wendet und die Gefäße unbeeinflußt läßt. Im gleichen Sinne hat sich kürzlich auch 0 . Müller ${ }^{1}$ ) ausgesprochen, und $O$. Loewi ${ }^{2}$ ) hat sogar eine gefäßdilatierende Wirkung von Strophantin und Digalen an den Nierenarterien nachgewiesen.

Die beginstigende Wirkung der Digitalis auf die Aorteninsuffzienz kommt also lediglich durch Erhöhung des Minimaldruckes zustande; dementsprechend war auch das Ausfließen aus der Carotis viel weniger intermittierend, als vorher. Daraus kann nun geschlossen werden, daß der Ventrikel in der Diastole weniger stark ausgedehnt wird, als vorher, und dieses Moment dürfte wohl die Erklärung abgeben für die von Cloëtta beobachteten Gewichtsdifferenzen bei Insuffizienzen mit und ohne ehronische Digitaliszufuhr. Durch welche Änderungen im feineren Mechanismus der Herzmuskeltätigkeit aber diese Verbesserung zustande kommt, das war mir zu eruieren unmöglich. Man kann an die Möglichkeit denken, daß die Muskelfasern der Aortenwurzel etwas anders arbeiten, sodaß hierdurch eine funktionelle Verbesserung der anatomischen Veränderung herbeigeführt wird, d. h. weniger Blut zuriickströmen kann.

\section{$\mathrm{Z}$ us a m menfassung.}

Die bei Kaninchen, Katzen und Hunden erzeugte Aorteninsuffizienz ergibt folgende Veränderungen des Kreislaufes:

1) 0. Müller, Verhandlg. d. Congr. f. inn. Med. 09.

2) O. Loewi, Arch. f. Experiment. Path. und Pharm. Bd. 59. 
Zur experimentellen Patbologie u. Therapie der akuten Aorteninsuffizienz. 209

Ganz konstant eine erhebliche Zunahme der Amplitude (120 Proz.), welche bedingt ist durch das regelmäßige beträchtliche Absinken des diastolischen Druckes (38 Proz.).

Der Maximaldruck bleibt meist unverändert, in einigen Fällen nimmt er $\mathrm{zu}$, in seltenern $\mathrm{ab}$.

Die diastolische Füllung des Herzens nimmt etwas zu, das Schlagvolumen verändert sich nicht nachweisbar.

Das kontinuierliehe Fließen in der Carotis wird in hochgradigen Fällen direkt intermittierend, die Ausflußmenge nimmt um 23,3 Proz. ab

Dureh Digitalis in therapeutisohen Dosen wird der Maximaldruck nicht verändert, der diastolische steigt um 17 Proz., die Amplitude verkleinert sich dementprechend um 12 Proz., die Ausflußmenge erhöht sich um 12 Proz.

Diese Veränderungen scheinen auschließlich durch Herzwirkung und nicht durch Gefäßverengerung zustande zu kommen. 